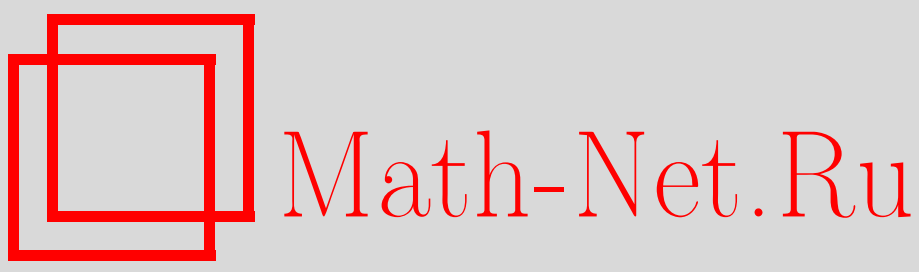

И. А. Васин, В. Л. Камынин, Асимптотическое поведение решений обратных задач для параболических уравнений с нерегулярными коэффициентами, Матем. сб., 1997, том 188, номер 3, 49-64

DOI: https://doi.org/10.4213/sm210

Использование Общероссийского математического портала Math-Net.Ru подразумевает, что вы прочитали и согласны с пользовательским соглашением

http://www.mathnet.ru/rus/agreement

Параметры загрузки:

IP: 3.85 .183 .62

26 апреля 2023 г., 11:30:59 


\section{Асимптотическое поведение решений обратных задач для параболических уравнений с нерегулярными коэффициентами}

Изучается вопрос об асимптотической при больших временах близости решений обратной задачи для параболического уравнения с неизвестной правой частью и решения предельной стационарной обратной задачи в ограниченной области. Условия переопределения задаются в интегральной форме. Требования близости коэффициентов уравнений в нестационарной и стационарной задачах задается в некотором весьма слабом смысле, допускающем, в частности, осциллирование этих коэффиициентов.

Библиография: 14 названий.

\section{§1. Введение}

В работе изучается вопрос об асимптотической при $t \rightarrow \infty$ близости решений $\{u(t, x), f(t)\}$ эволюционной обратной задачи

$$
\begin{gathered}
u_{t}-\sum_{i, j=1}^{n} \frac{\partial}{\partial x_{j}}\left(a_{i j}(t, x) u_{x_{i}}\right)+\sum_{i=1}^{n} b_{i}(t, x) u_{x_{i}}+\alpha u \\
=f(t) g(t, x)+h_{0}(t, x)+\sum_{j=1}^{n} \frac{\partial h_{j}(t, x)}{\partial x_{j}}, \quad(t, x) \in Q_{\infty} \\
u(0, x)=u_{0}(x), \quad x \in \Omega, \\
u(t, x)=0, \quad(t, x) \in[0,+\infty) \times \partial \Omega \\
\int_{\Omega} u(t, x) \eta(x) d x=\varphi(t), \quad t \in[0,+\infty)
\end{gathered}
$$

и решения $\{v(x), q\}$ стационарной обратной задачи

$$
\begin{gathered}
-\sum_{i, j=1}^{n} \frac{\partial}{\partial x_{j}}\left(a_{i j}^{\infty}(x) v_{x_{i}}\right)+\sum_{i=1}^{n} b_{i}^{\infty}(x) v_{x_{i}}+\alpha v \\
=q g^{\infty}(x)+h_{0}^{\infty}(x)+\sum_{j=1}^{n} \frac{\partial h_{j}^{\infty}(x)}{\partial x_{j}}, \quad x \in \Omega, \\
v(x)=0, \quad x \in \partial \Omega \\
\int_{\Omega} v(x) \eta(x) d x=\varphi^{\infty} .
\end{gathered}
$$

Работа вьполнена при частичной финансовой поддержке Российского фонда фундаментальных исследований (грант № 94-01-00289) и фонда ИНТАС (грант № 94-2187). 
Здесь $Q_{\infty} \equiv(0,+\infty) \times \Omega, \Omega$ - ограниченная область в $\mathbb{R}^{n}$ с гладкой границей. В обратной задаче (1.1)-(1.4) функции $a_{i j}(t, x), b_{i}(t, x), g(t, x), h_{j}(t, x), \eta(x), \varphi(t)$, $u_{0}(x)$ и константа $\alpha$ заданы, а пара функций $\{u(t, x), f(t)\}$ является искомой. В обратной задаче (1.5)-(1.7) функции $a_{i j}^{\infty}(x), b_{i}^{\infty}(x), g^{\infty}(x), h_{j}^{\infty}(x), \eta(x)$ и числа $\alpha$, $\varphi^{\infty}$ заданы, а функция $v(x)$ и константа $q$ подлежат определению.

Дополнительная информация о решении в рассматриваемых обратных задачах дается в виде условий интегрального переопределения (1.4) и (1.7), соответственно, что с физической точки зрения может, например, означать измерение температуры $u(t, x)$ и $v(x)$ с помошью датчика, производящего усреднение по области пространственных переменных $\Omega$.

Отметим, что обратная задача для параболического уравнения с интегральньм переопределением (1.4) и вопросы ее однозначной разрешимости изучались многими авторами (см., например, [1]-[4] и др.). Теорема существования и единственности решения задачи (1.5)-(1.7) в виде, достаточном для целей настояшей работы, установлена в $\S 2$. Она имеет также и самостоятельный интерес.

Теорема о близости решений задач (1.1)-(1.4) и (1.5)-(1.7) доказывается при условии близости соответствующих коэффициентов уравнений (1.1) и (1.5) в некотором слабом смысле: накладываемые условия допускают, в частности, осциллирующие коэффициенты в уравнении (1.1). При более жестких ограничениях на исходные данные асимптотическое при $t \rightarrow+\infty$ поведение решений обратных задач вида (1.1)-(1.4) исследовано ранее в [5].

Заметим, что в случае прямых задач для параболических уравнений в ограниченной области вопросы асимптотического поведения решений при больших временах широко изучены при различных предположениях относительно исходных данных; укажем здесь обзорные работы [6]-[9], где имеются дальнейшие ссылки. Кроме того, результаты (для прямых задач) при близких к предлагаемьм в настоящей работе предположениях относительно коэффициентов рассматриваемых уравнений были получены в [10], [11].

В случае же обратных задач проблема асимптотического поведения решений при больших временах ранее практически не исследовалась. Помимо упомянутой вьше работы [5] авторам известна лишь еще одна недавняя работа [12], где имеется результат по стабилизации решения обратной задачи в одном специальном частном случае.

Введем некоторые обозначения, используемые в дальнейшем. Положим $Q_{T}=(0, T] \times \Omega, Q_{t_{2}}^{t_{1}}=\left(t_{1}, t_{2}\right] \times \Omega, Q_{\infty}^{T}=(T,+\infty) \times \Omega$,

$$
G(t)=\int_{\Omega} g(t, x) \eta(x) d x, \quad G^{\infty}=\int_{\Omega} g^{\infty}(x) \eta(x) d x .
$$

Обозначение функциональных пространств $L_{2}(\Omega), L_{2}\left(Q_{t_{2}}^{t_{1}}\right)$,

$$
\begin{aligned}
& L_{r}\left(t_{1}, t_{2} ; L_{2}(\Omega)\right), \quad \stackrel{\circ}{W}{ }_{2}^{1}(\Omega), \quad W_{2}^{1}([0, T]), \quad C\left(0, T ; L_{2}(\Omega)\right), \\
& L_{2}\left(t_{1}, t_{2} ; \stackrel{\circ}{W} \underset{2}{1}(\Omega)\right), \quad W_{2}^{-1}(\Omega), \quad L_{2}\left(t_{1}, t_{2} ; W_{2}^{-1}(\Omega)\right), \quad C^{0, \gamma}\left(Q_{t_{2}}^{t_{1}}\right)
\end{aligned}
$$


с соответствующими нормами будем понимать в общепринятом смысле. Положим

$$
\begin{aligned}
& \|v\| \equiv\|v\|_{L_{2}(\Omega)}, \quad v(x) \in L_{2}(\Omega) ; \\
& \|\sigma\|_{Q_{T}}=\sup _{t \in[0, T]}\|\sigma(t, \cdot)\|+\left\|\sigma_{x}\right\|_{L_{2}\left(Q_{T}\right)}+\left\|\sigma_{t}\right\|_{L_{2}\left(0, T ; W_{2}^{-1}(\Omega)\right)}, \quad \sigma(t, x) \in C^{\infty}\left(Q_{T}\right) .
\end{aligned}
$$

Через ж обозначим константу из неравенства Пуанкаре-Стеклова

$$
\|u\| \leqslant \varkappa\left\|u_{x}\right\|
$$

которое справедливо для любой $u(x) \in \stackrel{\circ}{W} \underset{2}{1}(\Omega)$. Известно, что $\varkappa \equiv \varkappa(\Omega, n)>0$. Здесь

$$
\left\|u_{x}\right\|=\left(\int_{\Omega} \sum_{i=1}^{n} u_{x_{i}}^{2} d x\right)^{1 / 2} .
$$

Во всех дальнейших рассуждениях мы будем предполагать, что функции, входящие в исходные данные обратных задач, измеримы и удовлетворяют следующим условиям:

А) для любых $i, j=1,2, \ldots, n a_{i j}=a_{j i}, a_{i j}^{\infty}=a_{j i}^{\infty}$ и для любых $(t, x) \in Q_{\infty}$

$\Lambda_{0}|\xi|^{2} \leqslant \sum_{i, j=1}^{n} a_{i j}(t, x) \xi_{i} \xi_{j}, \quad \sum_{i, j=1}^{n} a_{i j}^{\infty}(x) \xi_{i} \xi_{j} \leqslant \Lambda_{1}|\xi|^{2} \quad \forall \xi=\left(\xi_{1}, \ldots, \xi_{n}\right) \in \mathbb{R}^{n} ;$

В) $\eta(x) \in \stackrel{\circ}{W} \frac{1}{2}(\Omega)$, причем $\|\eta\|_{W_{2}^{1}(\Omega)} \leqslant K_{1}$;

C) $\|g\|_{L_{\infty}\left(0, \infty ; L_{2}(\Omega)\right)},\left\|g^{\infty}\right\|,\left\|h_{j}^{\infty}\right\| \leqslant K_{2}, j=0,1,2, \ldots, n$;

$$
\left|\int_{\Omega} g(t, x) \eta(x) d x\right|,\left|\int_{\Omega} g^{\infty}(x) \eta(x) d x\right| \geqslant g_{0}, \quad t \in[0,+\infty) ;
$$

D) $\left|b_{i}(t, x)\right|,\left|b_{i}^{\infty}(x)\right| \leqslant K_{3}, i=1,2, \ldots, n,(t, x) \in Q_{\infty}$;

Е) $u_{0}(x) \in L_{2}(\Omega), \varphi(t) \in W_{2}^{1}([0, T])$ для любого $T>0$,

$$
\int_{\Omega} u_{0}(x) \eta(x) d x=\varphi(0)
$$

F) $\beta \equiv \alpha+\frac{\Lambda_{0}}{2 \varkappa^{2}}-\frac{2 n}{\Lambda_{0}}\left[K_{3}^{2}+n\left(\frac{\Lambda_{1} K_{1} K_{2}}{g_{0}}\right)^{2}+\left(\frac{K_{1} K_{2} K_{3}}{g_{0}}\right)^{2}\right]>0$;

G) $h_{j}(t, x) \in L_{2}\left(Q_{T}\right)$ для любого $T>0, j=0,1,2, \ldots, n$ и

$$
\sup _{m=0,1,2, \ldots}\left[\int_{m}^{m+1}|\varphi(t)|^{2} d t+\int_{m}^{m+1}\left|\varphi^{\prime}(t)\right|^{2} d t+\sum_{j=0}^{n} \int_{m}^{m+1}\left\|h_{j}(t, \cdot)\right\|^{2} d t\right] \leqslant K_{4} .
$$

Здесь $\Lambda_{0}, \Lambda_{1}, g_{0}, K_{1}, K_{2}, K_{3}, K_{4}=$ const $>0$.

Ниже через $c$ (возможно, с индексом) будем обозначать любые положительные константы, зависящие только от $n, \Lambda_{0}, \Lambda_{1}, g_{0}, K_{1}, K_{2}, K_{3}, K_{4}, \alpha, \varkappa, \varphi^{\infty}, \Omega,\left\|u_{0}\right\|$. В разных местах одной буквой обозначаются, вообше говоря, разные константы. 


\section{§ 2. Некоторые вспомогательные предложения и оценки}

Рассмотрим обратную задачу (1.1)-(1.4).

ОПРЕДЕЛЕНИЕ 1. Обобщеннбцм решением обратной задачи (1.1)-(1.4) назовем пару функций $\{u(t, x), f(t)\}$ таких, что

$$
\begin{gathered}
u \in C\left(0, T ; L_{2}(\Omega)\right) \cap L_{2}\left(0, T ; \stackrel{\circ}{W_{2}^{1}}(\Omega)\right), \\
u_{t} \in L_{2}\left(0, T ; W_{2}^{-1}(\Omega)\right), \quad f \in L_{2}([0, T]) \quad \forall T>0 ;
\end{gathered}
$$

эти функции удовлетворяют интегральному тождеству

$$
\begin{aligned}
& \frac{d}{d t} \int_{\Omega} u(t, x) \psi(x) d x+\sum_{i, j=1}^{n} \int_{\Omega} a_{i j}(t, x) u_{x_{i}}(t, x) \psi_{x_{j}}(x) d x \\
& \quad+\sum_{i=1}^{n} \int_{\Omega} b_{i}(t, x) u_{x_{i}}(t, x) \psi(x) d x+\alpha \int_{\Omega} u(t, x) \psi(x) d x \\
& \quad=f(t) \int_{\Omega} g(t, x) \psi(x) d x+\int_{\Omega} h_{0}(t, x) \psi(x) d x-\sum_{j=1}^{n} \int_{\Omega} h_{j}(t, x) \psi_{x_{j}}(x) d x
\end{aligned}
$$

которое справедливо как равенство в $L_{2}([0, T])$ (для любого $T>0$ ) при любой функции $\psi(x) \in \stackrel{\circ}{W} \frac{1}{2}(\Omega)$; кроме того, функция $u(t, x)$ удовлетворяет при всех $t \in[0,+\infty)$ соотношению (1.4).

Условия, достаточные для однозначной разрешимости обратной задачи (1.1)-(1.4), и некоторые нужные нам оценки устанавливает следующее утверждение.

Теорема 1. Пусть выполнены условия A)-G). Тогда решение $\{u, f\}$ обратной задачи (1.1)-(1.4) существует, единственно и удовлетворяет оченкам

$$
\begin{gathered}
\|u(t, \cdot)\|^{2} \leqslant \exp (-\beta t)\left\|u_{0}\right\|^{2} \\
+c_{1} \sup _{m=0,1,2, \ldots}\left\{\int_{m}^{m+1}\left[|\varphi(\tau)|^{2}+\left|\varphi^{\prime}(\tau)\right|^{2}+\sum_{j=0}^{n}\left\|h_{j}(\tau, \cdot)\right\|^{2}\right] d \tau\right\} \\
t \in(0,+\infty), \\
\sup _{m=0,1,2, \ldots}\left\{\int_{m}^{m+1}\left\|u_{x}(\tau, \cdot)\right\|^{2} d \tau\right\} \leqslant c_{2} \\
\sup _{m=0,1,2, \ldots}\left\{\int_{m}^{m+1}|f(\tau)|^{2} d \tau\right\} \leqslant c_{3}
\end{gathered}
$$

ДокАЗАТЕльство. Поскольку на исходные данныеобратной задачи (1.1)-(1.4) наложены условия А)-E), G), то обобщенное решение $\{u(t, x), f(t)\}$ этой задачи сушествует и единственно (см., например, [4]). Перейдем к выводу оценок (2.2)-(2.4). 
Полагая в интегральном тождестве $(2.1) \psi(x)=\eta(x)$ и учитывая переопределение (1.4), выводим для $f(t)$ соотношение

$$
\begin{gathered}
f(t)=\frac{1}{G(t)}\left[\int_{\Omega} \sum_{i, j=1}^{n} a_{i j}(t, x) u_{x_{i}} \eta_{x_{j}} d x+\int_{\Omega} \sum_{i=1}^{n} b_{i}(t, x) u_{x_{i}} \eta d x\right. \\
\left.-\int_{\Omega} h_{0}(t, x) \eta d x+\int_{\Omega} \sum_{j=1}^{n} h_{j}(t, x) \eta_{x_{j}} d x+\alpha \varphi(t)+\varphi^{\prime}(t)\right], \\
t \in(0,+\infty) .
\end{gathered}
$$

Подставив $(2.5)$ в (1.1), получим для функции $u(t, x)$ интегродифференциальное уравнение

$$
\begin{aligned}
u_{t}- & \sum_{i, j=1}^{n} \frac{\partial}{\partial x_{j}}\left(a_{i j}(t, x) u_{x_{i}}\right)+\sum_{i=1}^{n} b_{i}(t, x) u_{x_{i}}+\alpha u \\
= & \frac{g(t, x)}{G(t)}\left[\int_{\Omega} \sum_{i, j=1}^{n} a_{i j}(t, x) u_{x_{i}} \eta_{x_{j}} d x+\int_{\Omega} \sum_{i=1}^{n} b_{i}(t, x) u_{x_{i}} \eta d x\right. \\
& \left.\quad-\int_{\Omega} h_{0}(t, x) \eta d x+\int_{\Omega} \sum_{j=1}^{n} h_{j}(t, x) \eta_{x_{j}} d x\right]+\frac{\alpha \varphi(t)+\varphi^{\prime}(t)}{G(t)} g(t, x) \\
& +h_{0}(t, x)+\sum_{j=1}^{n} \frac{\partial h_{j}(t, x)}{\partial x_{j}}, \quad(t, x) \in Q_{\infty},
\end{aligned}
$$

которому эта функция удовлетворяет в смысле модифицированного интегрального тождества, получающегося из (2.1) после подстановки (2.5). Стандартные рассуждения, применяемые при выводе энергетического неравенства (см., например, [13, с. 164-169]), приводят к оценке

$$
\begin{aligned}
& \frac{1}{2} \frac{d}{d t}\left(\|u(t, \cdot)\|^{2}\right)+\Lambda_{0}\left\|u_{x}(t, \cdot)\right\|^{2}+\alpha\|u(t, \cdot)\|^{2} \\
& \leqslant \sqrt{n} K_{3}\|u(t, \cdot)\| \cdot\left\|u_{x}(t, \cdot)\right\| \\
&+\frac{K_{1} K_{2}}{g_{0}}\|u(t, \cdot)\|\left[n \Lambda_{1}\left\|u_{x}(t, \cdot)\right\|+\sqrt{n} K_{3}\left\|u_{x}(t, \cdot)\right\|+\sum_{j=0}^{n}\left\|h_{j}(t, \cdot)\right\|\right] \\
&+\frac{K_{2}}{g_{0}}\left[|\alpha||\varphi(t)|+\left|\varphi^{\prime}(t)\right|\right]\|u(t, \cdot)\|+\left\|h_{0}(t, \cdot)\right\|\|u(t, \cdot)\| \\
&+\left[\sum_{j=1}^{n}\left\|h_{j}(t, \cdot)\right\|^{2}\right]^{1 / 2}\left\|u_{x}(t, \cdot)\right\|, \quad t \in(0,+\infty) .
\end{aligned}
$$

Если воспользоваться неравенством Коши

$$
2|\xi \eta| \leqslant \varepsilon|\xi|^{2}+\varepsilon^{-1}|\eta|^{2}, \quad \varepsilon>0, \quad \xi, \eta \in \mathbb{R}^{1}
$$


и учесть неравенство (1.8), то из (2.7) следует, что

$$
\begin{gathered}
\frac{d}{d t}\left(\|u(t, \cdot)\|^{2}\right)+\beta\|u(t, \cdot)\|^{2} \leqslant c\left[|\varphi(t)|^{2}+\left|\varphi^{\prime}(t)\right|^{2}+\sum_{j=0}^{n}\left\|h_{j}(t, \cdot)\right\|^{2}\right], \\
t \in(0,+\infty) .
\end{gathered}
$$

Из (2.9) в силу леммы Гронуолла вытекает неравенство

$$
\begin{aligned}
\|u(t, \cdot)\|^{2} \leqslant & \exp (-\beta t)\left\|u_{0}\right\|^{2} \\
& +c \sup _{m=0,1,2, \ldots} \int_{m}^{m+1}\left[|\varphi(\tau)|^{2}+\left|\varphi^{\prime}(\tau)\right|^{2}+\sum_{j=0}^{n}\left\|h_{j}(\tau, \cdot)\right\|^{2}\right] d \tau \\
& \times \sum_{0 \leqslant m \leqslant t} \exp \{-\beta[t-(m+1)]\}, \quad t \in(0,+\infty),
\end{aligned}
$$

непосредственньм следствием которого и является оценка (2.2).

Для получения оценки (2.3) следует проинтегрировать соотношение (2.7) по $t$ в пределах от $m$ до $m+1$. Отбрасывая слагаемое $\frac{1}{2}\|u(m+1, \cdot)\|^{2}$ в левой части полученного неравенства, перенося член $\alpha\|u\|_{L_{2}\left(Q_{m+1}^{m}\right)}^{2}$ направо и преобразуя затем правую часть с помощью неравенства $(2.8)$, находим, что

$$
\begin{aligned}
\int_{m}^{m+1}\left\|u_{x}(\tau, \cdot)\right\|^{2} d \tau \leqslant & c_{1}\|u(m, \cdot)\|^{2}+c_{2} \int_{m}^{m+1}\|u(\tau, \cdot)\|^{2} d \tau \\
& +c_{3} \int_{m}^{m+1}\left[|\varphi(\tau)|^{2}+\left|\varphi^{\prime}(\tau)\right|^{2}+\sum_{j=0}^{n}\left\|h_{j}(\tau, \cdot)\right\|^{2}\right] d \tau
\end{aligned}
$$

Применяя уже доказанную оценку (2.2) и условие $\mathrm{G})$, из (2.11) получаем (2.3).

Наконец, оценка (2.4) является следствием формулы (2.5) и уже доказанных оценок (2.2) и (2.3). Теорема 1 доказана.

В дальнейших рассуждениях нам потребуются сведения о разрешимости в пространстве $\stackrel{\circ}{W} \frac{1}{2}(\Omega)$ следуюшей прямой задачи

$$
\begin{gathered}
-\sum_{i, j=1}^{n} \frac{\partial}{\partial x_{j}}\left(a_{i j}^{\infty}(x) z_{x_{i}}\right)+\sum_{i=1}^{n} b_{i}^{\infty}(x) z_{x_{i}}+\alpha z=h_{0}^{\infty}(x)+\sum_{j=1}^{n} \frac{\partial h_{j}^{\infty}(x)}{\partial x_{j}}, x \in \Omega, \\
z(x)=0, \quad x \in \partial \Omega
\end{gathered}
$$

ОПРЕДЕЛЕНИЕ 2. Под обобщенныц решением задачи (2.12)-(2.13) мы понимаем функцию $z(x) \in \stackrel{\circ}{W} \underset{2}{1}(\Omega)$, которая удовлетворяет интегральному тождеству

$$
\begin{gathered}
\int_{\Omega}\left[\sum_{i, j=1}^{n} a_{i j}^{\infty}(x) z_{x_{i}} \psi_{x_{j}}+\sum_{i=1}^{n} b_{i}^{\infty}(x) z_{x_{i}} \psi+\alpha z \psi\right] d x \\
=\int_{\Omega} h_{0}^{\infty}(x) \psi d x-\sum_{j=1}^{n} \int_{\Omega} h_{j}^{\infty}(x) \psi_{x_{j}} d x \\
\forall \psi(x) \in \stackrel{\circ}{W_{2}^{1}}(\Omega) .
\end{gathered}
$$

Справедлива 
ТЕОРема 2. Пусть выполнены условия A), С), D) и F). Тогда решение прямой задачи (2.12), (2.13) существует, единственно и удовлетворяет оценке

$$
\|z\|_{W_{2}^{1}(\Omega)}^{2} \leqslant c \sum_{j=0}^{n}\left\|h_{j}^{\infty}\right\|^{2} .
$$

ДокАЗАТЕЛЬСтво. Полагая в $(2.14) \psi(x)=z(x)$ и учитывая условия A), C), $\mathrm{D})$, получаем для любого возможного решения задачи $(2.12)-(2.13)$ неравенство

$$
\Lambda_{0}\left\|z_{x}\right\|^{2}+\alpha\|z\|^{2} \leqslant \sqrt{n} K_{3}\|z\| \cdot\left\|z_{x}\right\|+\left\|h_{0}^{\infty}\right\| \cdot\|z\|+\left(\sum_{j=1}^{n}\left\|h_{j}^{\infty}\right\|\right)\left\|z_{x}\right\| .
$$

Воспользовавшись для оценки первого и третьего слагаемых в правой части (2.16) неравенством (2.8) с $\varepsilon=\Lambda_{0} / 2$, а затем неравенством (1.8), находим, что

$$
\left(\frac{\Lambda_{0}}{2 \varkappa^{2}}+\alpha\right)\|z\|^{2} \leqslant \frac{n K_{3}^{2}}{\Lambda_{0}}\|z\|^{2}+\left\|h_{0}^{\infty}\right\| \cdot\|z\|+c_{1} \sum_{j=1}^{n}\left\|h_{j}^{\infty}\right\|^{2} .
$$

Из последнего неравенства с учетом условия F) получаем оценку

$$
\|z\|^{2} \leqslant c_{2} \sum_{j=0}^{n}\left\|h_{j}^{\infty}\right\|^{2}
$$

а тогда из (2.16) выводим также оценку

$$
\left\|z_{x}\right\|^{2} \leqslant c_{3} \sum_{j=0}^{n}\left\|h_{j}^{\infty}\right\|^{2}
$$

Из (2.17) и (2.18) вытекает (2.15) и единственность решения задачи (2.12)-(2.13). Но тогда, следуя доказательству теоремы 8.3 из [14, с. 174-176], получаем существование решения этой задачи. Теорема 2 доказана.

В дальнейшем через $p^{*}(x) \in \stackrel{\circ}{W} \underset{2}{1}(\Omega)$ обозначим обобщенное решение следующей прямой задачи

$$
\begin{gathered}
-\sum_{i, j=1}^{n} \frac{\partial}{\partial x_{j}}\left(a_{i j}^{\infty}(x) p_{x_{i}}^{*}\right)+\sum_{i=1}^{n} b_{i}^{\infty}(x) p_{x_{i}}^{*}+\alpha p^{*}=g^{\infty}(x), \quad x \in \Omega \\
p^{*}(x)=0, \quad x \in \partial \Omega
\end{gathered}
$$

Отметим, что согласно теореме 2 такое решение $p^{*}(x)$ существует, единственно и удовлетворяет оценке

$$
\left\|p^{*}\right\|_{W_{2}^{1}(\Omega)} \leqslant c .
$$

Перейдем к изучению обратной задачи (1.5)-(1.7). 
ОПРЕДЕЛЕНИЕ 3. Обобщенным решением задачи (1.5)-(1.7) называется пара $\{v(x), q\}$, состоящая из функции $v(x) \in \stackrel{\circ}{W} \underset{2}{1}(\Omega)$ и константы $q \in \mathbb{R}^{1}$ таких, что при любой $\psi(x) \in \stackrel{\circ}{W}_{2}^{1}(\Omega)$ справедливо интегральное тождество

$$
\begin{aligned}
\int_{\Omega}\left[\sum_{i, j=1}^{n} a_{i j}^{\infty}(x) v_{x_{i}} \psi_{x_{j}}+\sum_{i=1}^{n} b_{i}^{\infty}(x) v_{x_{i}} \psi+\alpha v \psi\right] d x \\
\quad=q \int_{\Omega} g^{\infty}(x) \psi d x+\int_{\Omega} h_{0}^{\infty}(x) \psi d x-\sum_{j=1}^{n} \int_{\Omega} h_{j}^{\infty}(x) \psi_{x_{j}} d x
\end{aligned}
$$

при этом функция $v(x)$ удовлетворяет также и соотношению (1.7).

ТЕОРЕМА 3. Пусть выполнены условия А)-D) и F). Предположим, что функиия $\eta(x)$ удовлетворяет условию

$$
\int_{\Omega} p^{*}(x) \eta(x) d x \neq 0 .
$$

Тогда обобщенное решение $\{v(x), q\}$ обратной задачи (1.5)-(1.7) существует, единственно и справедлива оценка

$$
\|v\|_{W_{2}^{1}(\Omega)} \leqslant c
$$

ДоказАтельство. Решение обратной задачи (1.5)-(1.7) будем искать в виде

$$
\{v(x), q\}=\{z(x), 0\}+\{p(x), q\}
$$

где функция $z(x)$ является обобщенным решением прямой задачи (2.12)-(2.13) (сушествование которого гарантировано теоремой 2), а пара $\{p(x), q\}$ является обобщенным решением обратной задачи

$$
\begin{gathered}
-\sum_{i, j=1}^{n} \frac{\partial}{\partial x_{j}}\left(a_{i j}^{\infty}(x) p_{x_{i}}\right)+\sum_{i=1}^{n} b_{i}^{\infty}(x) p_{x_{i}}+\alpha p=q g^{\infty}(x), \quad x \in \Omega \\
p(x)=0, \quad x \in \partial \Omega \\
\int_{\Omega} p(x) \eta(x) d x=\varphi^{\infty}-\int_{\Omega} z(x) \eta(x) d x \equiv \zeta^{\infty}
\end{gathered}
$$

Положим $\zeta^{*}=\int_{\Omega} p^{*}(x) \eta(x) d x$, где $p^{*}(x) \in \stackrel{\circ}{W} \underset{2}{1}(\Omega)$ - обобщенное решение задачи $(2.19),(2.20)$. В силу $(2.23) \zeta^{*} \neq 0$. Непосредственной подстановкой убеждаемся, что пара

$$
p(x) \equiv p^{*}(x) \zeta^{\infty} / \zeta^{*}, \quad q \equiv \zeta^{\infty} / \zeta^{*}
$$

является решением обратной задачи (2.26)-(2.28).

Тем самым, мы установили, что решение исходной обратной задачи (1.5)-(1.7) существует. 
Покажем, что оно единственно. Предположим, что сушествуют два решения $\left\{v_{1}(x), q_{1}\right\}$ и $\left\{v_{2}(x), q_{2}\right\}$ задачи (1.5)-(1.7). Тогда пара $\hat{p}(x) \equiv v_{1}(x)-v_{2}(x)$, $\hat{q} \equiv q_{1}-q_{2}$ удовлетворяет соотношениям

$$
\begin{gathered}
-\sum_{i, j=1}^{n} \frac{\partial}{\partial x_{j}}\left(a_{i j}^{\infty}(x) \hat{p}_{x_{i}}\right)+\sum_{i=1}^{n} b_{i}^{\infty}(x) \hat{p}_{x_{i}}+\alpha \hat{p}=\hat{q} g^{\infty}(x), \quad x \in \Omega \\
\hat{p}(x)=0, \quad x \in \partial \Omega \\
\int_{\Omega} \hat{p}(x) \eta(x) d x=0 .
\end{gathered}
$$

Сравнивая соотношения (2.19)-(2.20) и (2.30)-(2.31), убеждаемся, что функции $p^{*}(x)$ и $\hat{p}(x) / \hat{q}$ являются решениями одной и той же прямой задачи $(2.19),(2.20)$, и поэтому в силу единственности такого решения $\hat{p}(x) \equiv \hat{q} p^{*}(x)$. Но тогда в соответствии с (2.32)

$$
0=\int_{\Omega} \hat{p}(x) \eta(x) d x=\hat{q} \int_{\Omega} p^{*}(x) \eta(x) d x,
$$

откуда следует, что $\hat{q}=0$, ибо $p^{*}(x)$ и $\eta(x)$ удовлетворяют соотношению (2.23). Таким образом, $q_{1}=q_{2}$. Совпадение функций $v_{1}(x)$ и $v_{2}(x)$ вытекает теперь из единственности решения соответствуюшей прямой задачи (1.5)-(1.6).

Итак, решение обратной задачи (1.5)-(1.7) сушествует и единственно. Наконец, оценка (2.24) является следствием представления (2.25), формул (2.29) и оценок $(2.15),(2.21)$. Теорема 3 доказана.

ЗАмечАние 1. Условие (2.23) является необходимьм для единственности решения обратной задачи (1.5)-(1.7). Действительно, предположим, что

$$
\int_{\Omega} p^{*}(x) \eta(x) d x=0
$$

и пусть пара $\{v(x), q\}$ является обобшенным решением задачи (1.5)-(1.7). Тогда, очевидно, любая пара вида $\left\{v(x)+\theta p^{*}(x), q+\theta\right\}$ (где $p^{*}(x)$ - решение задачи $(2.19),(2.20)$, а $\theta$ - произвольная константа из $\left.\mathbb{R}^{1}\right)$ также является решением обратной задачи (1.5)-(1.7). Более того, если (2.23) не вьполнено, то решение задачи (1.5)-(1.7) сушествует не при всех исходных данных, удовлетворяюших условиям A)-D) и F). Необходимо еше, как нетрудно видеть, выполнение условия

$$
\int_{\Omega} z(x) \eta(x) d x=\varphi^{\infty}
$$

где $z(x)$ - решение прямой задачи $(2.12),(2.13)$.

\section{§3. Основной результат}

Всюду ниже будем использовать обозначения

$$
F^{k}(t, x) \equiv F(t+k, x), \quad F^{k}(t) \equiv F(t+k), \quad k=1,2, \ldots .
$$


Теорема 4. Пусть выполнены условия A)-G) и (2.23). Предположим, что при $k \rightarrow \infty$ слабо в $L_{2}\left(Q_{1}\right)$

$$
\begin{aligned}
a_{i j}^{k}(t, x) & \rightarrow a_{i j}^{\infty}(x), & i, j & =1,2, \ldots, n ; \\
b_{i}^{k}(t, x) & \rightarrow b_{i}^{\infty}(x), & i & =1,2, \ldots, n ; \\
h_{j}^{k}(t, x) & \rightarrow h_{j}^{\infty}(x), & j & =0,1,2, \ldots, n
\end{aligned}
$$

слабо в $L_{2}(\Omega)$ при кажсдом фиксированном $t \in[0,1]$

$$
g^{k}(t, x) \rightarrow g^{\infty}(x)
$$

в норме $L_{2}([0,1])$

$$
\varphi^{k}(t) \rightarrow \varphi^{\infty}
$$

Кроме того, пусть существует последовательность $\mu(k) \rightarrow 0, k \rightarrow \infty$, такая, что для любой функиии $\sigma(t, x) \in C^{\infty}\left(Q_{1}\right)$, равной нулю на боковой поверхности иилиндра $Q_{1}$, справедливо неравенство

$$
\begin{aligned}
\sum_{j=1}^{n} \mid \int_{Q_{1}} \sum_{i=1}^{n} & {\left[a_{i j}^{k}(t, x)-a_{i j}^{\infty}(x)\right] \sigma_{x_{i}}(t, x) d x d t \mid } \\
+ & \left|\int_{Q_{1}} \sum_{i=1}^{n}\left[b_{i}^{k}(t, x)-b_{i}^{\infty}(x)\right] \sigma_{x_{i}}(t, x) d x d t\right| \leqslant \mu(k)\|\sigma\|_{Q_{1}}
\end{aligned}
$$

Тогда при любой начальной функиии $u_{0}(x) \in L_{2}(\Omega)$ в условии (1.2) обобщенное решение $\{u(t, x), f(t)\}$ обратной задачи (1.1)-(1.4) сходится к решению $\{v(x), q\}$ обратной задачи (1.5)-(1.7) в следующем смысле:

$$
\begin{gathered}
\lim _{m \rightarrow \infty} \int_{m}^{m+1}\|u(t, \cdot)-v(\cdot)\|^{2} d t=0 \\
\lim _{m \rightarrow \infty} \int_{m}^{m+1} \int_{\Omega}\left[u_{x_{i}}(t, x)-v_{x_{i}}(x)\right] \psi(x) d x d t=0, \\
i=1,2, \ldots, n, \quad \forall \psi(x) \in L_{2}(\Omega) \\
\lim _{m \rightarrow \infty} \int_{m}^{m+1} f(t) d t=q .
\end{gathered}
$$

ЗАмечАниЕ 2. Условие (3.1) выполнено в цилиндре $Q_{N}$, а условия (3.2), (3.3) вьполнены на отрезке $[0, N]$ при любом $N=1,2, \ldots$ Условие (3.4) также выполнено в $Q_{N}$ при любом $N=1,2, \ldots$ с заменой нормы $\|\sigma\|_{Q_{1}}$ на норму $\|\sigma\|_{Q_{N}}$ и с некоторой другой бесконечно малой последовательностью $\mu_{N}(k)$ (зависящей от $N$ ) в правой части (3.4). Доказательство этих утверждений аналогично доказательству лемм 2.1 и 2.2 из [11]. 
ДоКАЗАТЕЛЬСТво ТЕОРЕМЫ 4. Фиксируем произвольное $\varepsilon>0$. Для каждого $k=1,2, \ldots$ представим решение $\{u(t, x), f(t)\}$ обратной задачи (1.1)-(1.4), рассматриваемой при $t \geqslant k$, в виде суммы

$$
\{u(t, x), f(t)\}=\left\{p^{(k)}(t, x), r^{(k)}(t)\right\}+\left\{w^{(k)}(t, x), s^{(k)}(t)\right\} .
$$

Здесь $\left\{w^{(k)}(t, x), s^{(k)}(t)\right\}$ есть решение в $Q_{\infty}^{k}$ обратной задачи

$$
\begin{gathered}
w_{t}^{(k)}-\sum_{i, j=1}^{n} \frac{\partial}{\partial x_{j}}\left(a_{i j}(t, x) w_{x_{i}}^{(k)}\right)+\sum_{i=1}^{n} b_{i}(t, x) w_{x_{i}}^{(k)}+\alpha w^{(k)}=s^{(k)}(t) g(t, x) \\
(t, x) \in Q_{\infty}^{k} \\
w^{(k)}(k, x)=u(k, x)-v(x), \quad x \in \Omega \\
w^{(k)}(t, x)=0, \quad(t, x) \in[k,+\infty) \times \partial \Omega \\
\int_{\Omega} w^{(k)}(t, x) \eta(x) d x=0, \quad t \in[k,+\infty)
\end{gathered}
$$

а через $\left\{p^{(k)}(t, x), r^{(k)}(t)\right\}$ обозначено решение в $Q_{\infty}^{k}$ обратной задачи

$$
\begin{gathered}
p_{t}^{(k)}-\sum_{i, j=1}^{n} \frac{\partial}{\partial x_{j}}\left(a_{i j}(t, x) p_{x_{i}}^{(k)}\right)+\sum_{i=1}^{n} b_{i}(t, x) p_{x_{i}}^{(k)}+\alpha p^{(k)} \\
=r^{(k)}(t) g(t, x)+h_{0}(t, x)+\sum_{j=1}^{n} \frac{\partial h_{j}(t, x)}{\partial x_{j}}, \quad(t, x) \in Q_{\infty}^{k}, \\
p^{(k)}(k, x)=v(x), \quad x \in \Omega, \\
p^{(k)}(t, x)=0, \quad(t, x) \in[k,+\infty) \times \partial \Omega, \\
\int_{\Omega} p^{(k)}(t, x) \eta(x) d x=\varphi(t), \quad t \in[k,+\infty) .
\end{gathered}
$$

Согласно теореме 1 решения этих обратных задач существуют и единственны. Рассмотрим задачу (3.9)-(3.12). В силу теорем 1 и 2

$$
\left\|w^{(k)}(t, \cdot)\right\| \leqslant\|u(k, \cdot)-v(\cdot)\| \exp \left[\frac{-\beta(t-k)}{2}\right] \leqslant c_{1} \exp \left[\frac{-\beta(t-k)}{2}\right],
$$

где константа $c_{1}>0$ не зависит от $t$ и $k$. Неравенство (3.17) позволяет подобрать такое положительное число $N_{1}(\varepsilon)$, что при любом $t \geqslant N_{1}(\varepsilon)+k$ будет справедлива оценка

$$
\left\|w^{(k)}(t, \cdot)\right\|<\varepsilon .
$$

Запишем неравенство (2.11) применительно к функции $w^{(k)}(t, x)$ и воспользуемся оценкой (3.17). В результате получим

$$
\int_{m}^{m+1}\left\|w_{x}^{(k)}(t, \cdot)\right\|^{2} d t \leqslant c_{2} \exp [-\beta(m-k)]
$$


где константа $c_{2}>0$ не зависит от $m$ и $k$. Следовательно, сушествует положительное число $N_{2}(\varepsilon)$ такое, что при любом $m \geqslant N_{2}(\varepsilon)+k$ имеет место оценка

$$
\int_{m}^{m+1}\left\|w_{x}^{(k)}(t, \cdot)\right\|^{2} d t<\varepsilon
$$

Рассмотрим теперь функцию $s^{(k)}(t)$. Соотношение типа $(2.5)$, записанное для $s^{(k)}(t)$, выглядит так:

$$
s^{(k)}(t)=\frac{1}{G(t)}\left[\int_{\Omega} \sum_{i, j=1}^{n} a_{i j}(t, x) w_{x_{i}}^{(k)} \eta_{x_{j}} d x+\int_{\Omega} \sum_{i=1}^{n} b_{i}(t, x) w_{x_{i}}^{(k)} \eta d x\right] .
$$

Тогда из (3.21) с учетом (3.19) получим

$$
\int_{m}^{m+1}\left|s^{(k)}(t)\right|^{2} d t \leqslant c_{3} \int_{m}^{m+1}\left\|w_{x}^{(k)}(t, \cdot)\right\|^{2} d t \leqslant c_{4} \exp [-\beta(t-k)],
$$

где $c_{4}=$ const $>0$ не зависит от $m$ и $k$. Исходя из оценки (3.22), можно выбрать такое положительное число $N_{3}(\varepsilon)$, что при $m \geqslant N_{3}(\varepsilon)+k$

$$
\int_{m}^{m+1}\left|s^{(k)}(t)\right|^{2} d t<\varepsilon
$$

Положим

$$
N=\max \left\{N_{1}(\varepsilon), N_{2}(\varepsilon), N_{3}(\varepsilon)\right\}+1
$$

и рассмотрим задачу (3.13)-(3.16) в цилиндре $Q_{k+N}^{k}$. Сделав замену $t=\tau+k$, введем следующие обозначения:

$$
z^{(k)}(\tau, x)=p^{(k)}(\tau+k, x) \equiv p^{(k)}(t, x), \quad y^{(k)}(\tau)=r^{(k)}(\tau+k) \equiv r^{(k)}(t)
$$

Тогда пара функций $\left\{z^{(k)}(\tau, x), y^{(k)}(\tau)\right\}$ является в $Q_{N}$ решением обратной задачи

$$
\begin{gathered}
z_{\tau}^{(k)}-\sum_{i, j=1}^{n} \frac{\partial}{\partial x_{j}}\left(a_{i j}^{k}(\tau, x) z_{x_{i}}^{(k)}\right)+\sum_{i=1}^{n} b_{i}^{k}(\tau, x) z_{x_{i}}^{(k)}+\alpha z^{(k)} \\
=y^{(k)}(\tau) g^{k}(\tau, x)+h_{0}^{k}(\tau, x)+\sum_{j=1}^{n} \frac{\partial h_{j}^{k}(\tau, x)}{\partial x_{j}}, \quad(\tau, x) \in Q_{N} \\
z^{(k)}(0, x)=v(x), \quad x \in \Omega, \\
\int_{\Omega}^{(k)}(\tau, x)=0, \quad(\tau, x) \in[0, N] \times \partial \Omega \\
\int^{(k)}(\tau, x) \eta(x) d x=\varphi^{k}(\tau), \quad \tau \in[0, N] .
\end{gathered}
$$

В силу теоремы 1 с учетом (2.24) и условия $\mathrm{G}$ ) справедливы следуюшие равномерные относительно $k$ оценки

$$
\begin{gathered}
\sup _{\tau \in[0, N]}\left\|z^{(k)}(\tau, \cdot)\right\| \leqslant c_{1}, \\
\left\|z_{x}^{(k)}\right\|_{L_{2}\left(Q_{N}\right)} \leqslant c_{2}(N), \\
\left\|y^{(k)}\right\|_{L_{2}([0, N])} \leqslant c_{3}(N) .
\end{gathered}
$$


Кроме того, из результатов работы [4] вытекает равномерная по $k$ оценка

$$
\left\|z_{\tau}^{(k)}\right\|_{L_{2}\left(0, N ; W_{2}^{-1}(\Omega)\right)} \leqslant c_{4}(N) .
$$

Согласно (3.30)-(3.33) найдутся подпоследовательность $k_{\nu} \rightarrow \infty$, функции $z^{(\infty)}(\tau, x) \in C\left(0, N ; L_{2}(\Omega)\right) \cap L_{2}(0, N ; \stackrel{\circ}{W} \underset{2}{1}(\Omega)), z_{\tau}^{(\infty)}(\tau, x) \in L_{2}\left(0, N ; W_{2}^{-1}(\Omega)\right)$, а также функция $y^{(\infty)}(\tau) \in L_{2}([0, N])$ такие, что при $\nu \rightarrow \infty$ в норме $L_{2}\left(Q_{N}\right)$

$$
z^{\left(k_{\nu}\right)}(\tau, x) \rightarrow z^{(\infty)}(\tau, x)
$$

слабо в $L_{2}\left(Q_{N}\right)$

$$
z_{x}^{\left(k_{\nu}\right)}(\tau, x) \rightarrow z_{x}^{(\infty)}(\tau, x)
$$

слабо в $L_{2}\left(0, N ; W_{2}^{-1}(\Omega)\right)$

$$
z_{\tau}^{\left(k_{\nu}\right)}(\tau, x) \rightarrow z_{\tau}^{(\infty)}(\tau, x)
$$

слабо в $L_{2}([0, N])$

$$
y^{\left(k_{\nu}\right)}(\tau) \rightarrow y^{(\infty)}(\tau) .
$$

В силу условий $(3.1)-(3.4)$ к задаче $\left(3.26^{k}\right)-\left(3.29^{k}\right)$ можно применить теорему 2 работы [4], согласно которой пара $\left\{z^{(\infty)}(\tau, x), y^{(\infty)}(\tau)\right\}$ является обобщенным решением в $Q_{N}$ обратной задачи

$$
\begin{gathered}
z_{\tau}^{(\infty)}-\sum_{i, j=1}^{n} \frac{\partial}{\partial x_{j}}\left(a_{i j}^{\infty}(x) z_{x_{i}}^{(\infty)}\right)+\sum_{i=1}^{n} b_{i}^{\infty}(x) z_{x_{i}}^{(\infty)}+\alpha z^{(\infty)} \\
=y^{(\infty)}(\tau) g^{\infty}(x)+h_{0}^{\infty}(x)+\sum_{j=1}^{n} \frac{\partial h_{j}^{\infty}(x)}{\partial x_{j}}, \quad(\tau, x) \in Q_{N}, \\
z^{(\infty)}(0, x)=v(x), \quad x \in \Omega, \\
z^{(\infty)}(\tau, x)=0, \quad(\tau, x) \in[0, N] \times \partial \Omega \\
\int_{\Omega} z^{(\infty)}(\tau, x) \eta(x) d x=\varphi^{\infty} .
\end{gathered}
$$

С другой стороны, пара $\{v(x), q\}$, очевидно, также является обобшенным решением этой задачи в $Q_{N}$. В силу единственности обобшенного решения задачи (3.38)-(3.41) (см. [4]) имеем

$$
z^{(\infty)}(\tau, x) \equiv v(x), \quad y^{(\infty)}(\tau) \equiv q,
$$

и, кроме того, сходимость в (3.34)-(3.37) осушествляется по всей последовательности индексов $k \rightarrow \infty$.

Следовательно, в силу (3.34) мы можем указать такой номер $k_{1}(\varepsilon)$, что при любом $k \geqslant k_{1}(\varepsilon)$

$$
\int_{0}^{N} \int_{\Omega}\left|z^{(k)}(\tau, x)-v(x)\right|^{2} d x d \tau \equiv \int_{k}^{k+N}\left\|p^{(k)}(t, \cdot)-v(\cdot)\right\|^{2} d t<\varepsilon .
$$


Заметим, что

$$
\begin{aligned}
& \int_{k+N-1}^{k+N}\|u(t, \cdot)-v(\cdot)\|^{2} d t \\
& \quad \leqslant 2 \int_{k+N-1}^{k+N}\left\|w^{(k)}(t, \cdot)\right\|^{2} d t+2 \int_{k+N-1}^{k+N}\left\|p^{(k)}(t, \cdot)-v(\cdot)\right\|^{2} d t .
\end{aligned}
$$

Пусть $k \geqslant k_{1}(\varepsilon)$. Тогда из (3.43) следует, что второе слагаемое в правой части (3.44) будет меньше $2 \varepsilon$. Поскольку в силу $(3.24) N-1 \geqslant N_{1}(\varepsilon)$, то первое слагаемое в правой части (3.44) будет меньше $2 \varepsilon^{2}$ согласно (3.18).

Таким образом, для любого $\varepsilon>0$ сушествует $N_{4}(\varepsilon) \equiv k_{1}(\varepsilon)+N-1$ такое, что при $m \geqslant N_{4}(\varepsilon)$

$$
\int_{m}^{m+1}\|u(t, \cdot)-v(\cdot)\|^{2} d t \leqslant 2\left(\varepsilon+\varepsilon^{2}\right)
$$

откуда и вытекает (3.5).

Аналогично, в силу $(3.35)$ для любой $\psi(x) \in L_{2}(\Omega)$ найдется $k_{2}(\varepsilon)$ такое, что для любого $i=1,2, \ldots, n$ при $k \geqslant k_{2}(\varepsilon)$

и потому

$$
\left|\int_{N-1}^{N} \int_{\Omega}\left[z_{x_{i}}^{(k)}(\tau, x)-v_{x_{i}}(x)\right] \psi(x) d x d \tau\right|<\varepsilon,
$$

$$
\left|\int_{k+N-1}^{k+N} \int_{\Omega}\left[p_{x_{i}}^{(k)}(t, x)-v_{x_{i}}(x)\right] \psi(x) d x d t\right|<\varepsilon .
$$

Но тогда при $k \geqslant k_{2}(\varepsilon)$ с учетом (3.20), (3.45) и (3.24) получаем оценку

$$
\begin{aligned}
& \left|\int_{k+N-1}^{k+N} \int_{\Omega}\left[u_{x_{i}}(t, x)-v_{x_{i}}(x)\right] \psi(x) d x d t\right| \\
& \quad \leqslant c(\psi) \int_{k+N-1}^{k+N}\left\|w_{x}^{(k)}(t, \cdot)\right\| d t+\left|\int_{k+N-1}^{k+N} \int_{\Omega}\left[p_{x_{i}}^{(k)}(t, x)-v_{x_{i}}(x)\right] \psi(x) d x d t\right| \\
& \quad \leqslant \sqrt{\varepsilon} c(\psi)+\varepsilon
\end{aligned}
$$

из которой, очевидно, следует (3.6).

Пусть, наконец, $\chi_{[N-1, N]}(\tau)$ - характеристическая функция отрезка $[N-1, N]$. В силу (3.37) найдется число $k_{3}(\varepsilon)$ такое, что для любого $k \geqslant k_{3}(\varepsilon)$

$$
\left|\int_{0}^{N}\left[y^{(k)}(\tau)-q\right] \chi_{[N-1, N]}(\tau) d \tau\right| \equiv\left|\int_{N-1}^{N}\left[y^{(k)}(\tau)-q\right] d \tau\right|<\varepsilon
$$

или в другой записи (см. (3.25))

$$
\left|\int_{k+N-1}^{k+N}\left[r^{(k)}(t)-q\right] d t\right|<\varepsilon
$$

Тогда при $k \geqslant k_{3}(\varepsilon)$, учитывая (3.23), (3.46) и (3.24), приходим к оценке

$$
\begin{gathered}
\left|\int_{k+N-1}^{k+N}[f(t)-q] d t\right| \leqslant \int_{k+N-1}^{k+N}\left|s^{(k)}(t)\right| d t+\left|\int_{k+N-1}^{k+N}\left[r^{(k)}(t)-q\right] d t\right| \\
\quad \leqslant\left\{\int_{k+N-1}^{k+N}\left|s^{(k)}(t)\right|^{2} d t\right\}^{1 / 2}+\left|\int_{k+N-1}^{k+N}\left[r^{(k)}(t)-q\right] d t\right| \leqslant \sqrt{\varepsilon}+\varepsilon,
\end{gathered}
$$

откуда вытекает (3.7). Теорема 4 доказана. 
ЗАмЕчАнИЕ 3 . В теореме 4 близость $f(t)$ и $q$ удается доказать только в достаточно слабом смысле (см. соотношение (3.7)). Это связано с весьма слабыми предположениями о близости коэффициентов уравнений (1.1) и (1.5) в виде условий $(3.1),(3.2),(3.4)$. Если потребовать выполнения более сильных условий сходимости при $t \rightarrow \infty$ коэффициентов уравнения (1.1) к соответствуюшим коэффициентам уравнения (1.5), то можно доказать и сходимость $f(t)$ к $q$ в более сильном смысле. Соответствующим результатам будет посвящена другая статья. Один из таких результатов в краткой форме анонсирован в [5].

В рамках данной работы, несколько усилив предположения о правых частях уравнений (1.1) и (1.5), получим ниже более сильное, нежели в теореме 4 , утверждение о близости функции $u(t, x)$ к $v(x)$. Именно, справедлива

ТЕОРема 5. Пусть в дополнение $к$ условиям теоремь 4 известно, ито

$$
|g(t, x)|,\left|g^{\infty}(x)\right|,\left|h_{j}(t, x)\right|,\left|h_{j}^{\infty}(t, x)\right| \leqslant K_{5},
$$

$j=0,1,2, \ldots, n ; \quad(t, x) \in Q_{\infty}, K_{5}=$ const $>0$. Тогда в теореме 4 вместо (3.5) будет выполнено соотношение

$$
\sup _{x \in \Omega}|u(t, x)-v(x)| \rightarrow 0, \quad t \rightarrow \infty .
$$

ДоКАЗАТЕЛЬСТво. Учитывая условие (3.47) и теорему 8.1 из [13, с. 225], примененную к уравнению (1.1), вьводим оценку

$$
\sup _{(t, x) \in Q_{m+1}^{m}}|u(t, x)| \leqslant c_{1}, \quad m=1,2, \ldots,
$$

где $c_{1}=$ const $>0$ зависит от $\Lambda_{0}, \Lambda_{1}, K_{2}, K_{3}, K_{5}, n, \alpha,\|u\|_{L_{2}\left(Q_{m+2}^{m-1}\right)}$ и $\|f\|_{L_{2}([m-1, m+2])}$. В свою очередь, согласно (2.2) и (2.4) получаем, что нормы $\|u\|_{L_{2}\left(Q_{m+2}^{m-1}\right)}$ и $\|f\|_{L_{2}([m-1, m+2])}$ оцениваются сверху константами, не зависящими от $m$. Поэтому константа $c_{1}$ в $(3.49)$ не зависит от $m$ и из (3.49) следует, очевидно, оценка

$$
\sup _{(t, x) \in Q_{\infty}}|u(t, x)| \leqslant c_{2},
$$

где $c_{2}=$ const $>0$ также не зависит от $m$.

В силу сделанных предположений и доказанной оценки (3.50) из теоремы 10.1 (см. [13, с. 238]) вытекает оценка

$$
|u|_{C^{0, \gamma}\left(Q_{m+1}^{m}\right)} \leqslant c_{3}, \quad m=1,2, \ldots
$$

Здесь $\gamma=$ const $\in(0,1)$ и $c_{3}=$ const $>0$ не зависят от $m$.

Рассмотрим последовательность $u^{k}(t, x), k=1,2, \ldots$, считая, что $(t, x) \in Q_{2}^{1}$. Напомним, что $u^{k}(t, x) \equiv u(t+k, x)$. В силу (3.51) эта последовательность является равномерно ограниченной и равностепенно непрерывной в цилиндре $Q_{2}^{1}$. Тогда согласно теореме Арцела найдется подпоследовательность $k_{\nu} \rightarrow \infty$ и функция $v_{1}(t, x)$ такие, что равномерно на $Q_{2}^{1}$ при $\nu \rightarrow \infty$

$$
u^{k_{\nu}}(t, x) \rightrightarrows v_{1}(t, x) .
$$


В то же время, учитьвая предельное соотношение $(3.5)$, имеем, что при $\nu \rightarrow \infty$

$$
\int_{1}^{2}\left\|u^{k_{\nu}}(t, \cdot)-v(\cdot)\right\|^{2} d t \equiv \int_{k_{\nu}+1}^{k_{\nu}+2}\|u(t, \cdot)-v(\cdot)\|^{2} d t \rightarrow 0 .
$$

Следовательно, $v_{1}(t, x) \equiv v(x)$ и, кроме того, сходимость в $(3.52)$ будет осушествляться по всей последовательности $k \rightarrow \infty$, т.е.

$$
\lim _{k \rightarrow \infty} \sup _{(t, x) \in Q_{2}^{1}}\left|u^{k}(t, x)-v(\cdot)\right|=0,
$$

что немедленно приводит к предельному соотношению (3.48).

Теорема 5 доказана.

\section{Список литературы}

1. Прилепко А.И., Орловский Д. Г. Об определении параметра эволюционного уравнения и обратных задачах математической физики. II // Дифференц. уравнения. 1985. T. 21. № 4. C. $694-700$.

2. Cannon J.R., Lin $Y$. Determination of a parameter $p(t)$ in some quasilinear parabolic differential equations // Inverse Problems. 1988. V. 4. № 1. P. 35-45.

3. Костин А.Б. Обратная задача для уравнения теплопроводности с интегральньгм переопределением // Обратные задачи для математического моделирования физических процессов. Сб. научных трудов. М.: МИФИ, 1991. С. 45-49.

4. Kamynin V.L. On convergence of the solutions of inverse problems for parabolic equations with weakly converging coefficients // Elliptic and Parabolic Problems, Pont-a-Mousson. 1994; // Pitman Res. Notes Math. Ser. 1995. V. 325. P. 130-151.

5. Васин И. А., Камынин В. Л. Об асимптотическом поведении слабых решений обратных задач для параболических уравнений // Успехи матем. наук. 1995. Т. 50. № 4. С. 144 .

6. Ильин A.M., Калашников A. С., Олейник O. А. Линейные уравнения второго порядка параболического типа // Успехи матем. наук. 1962. Т. 17. № 3. С. 3-146.

7. Зеленяк Т.И., Михайлов В. П. Асимптотическое поведение решений некоторых краевых задач математической физики при $t \rightarrow \infty / /$ Труды симпозиума, посвящ. 60-летию С. Л. Соболева. М.: Наука, 1970. С. 96-119.

8. Гущин A.K., Михайлов В. П., Муравей Л. А. О стабилизации решений нестационарных граничных задач для линейных дифференциальных уравнений в частных производных // Динамика сплошной среды. Т. 23. Новосибирск: Изд-во СОАН СССР, 1975. C. 57-91.

9. Вишневский М. П., Зеленяк Т.И., Лаврентьев М. М. Поведение решений нелинейных параболических уравнений при большом времени // Сибирский матем. журнал. 1995. T. 36. № 3. С. 510-530.

10. Arosio A. Asymptotic behavior as $t \rightarrow \infty$ of solutions of linear parabolic equations with discontinuous coefficients in a bounded domain // Comm. Part. Diff. Equations. 1979. V. 4. № 7 . P. 769-794.

11. Камынин В. Л. Асимптотическое поведение решений квазилинейных параболических уравнений в ограниченной области // Сибирский матем. журнал. 1994. Т. 35. № 2. C. 340-358.

12. Riganti R., Savateev E. Solution of an inverse problem for the nonlinear heat equation // Comm. Partial Differential Equations. 1994. V. 19. № 9, 10. P. 1611-1628.

13. Ладыженская О. А., Солонников В. А., Уральцева Н. Н. Линейные и квазилинейные уравнения параболического типа. М.: Наука, 1967.

14. Гилбарг Д., Трудингер Н. Эллиптические дифференциальные уравнения с частньми производными второго порядка. М.: Наука, 1989. 